\section{Economic aspects}

The average cost for one printed value, after automatic recording of the primary value, the calculation of a final result with a statistical evaluation and different outprints, is, at present, about 0.4 Swedish crowns. This represents about ten per cent of the average cost

\begin{tabular}{|c|c|c|}
\hline \multicolumn{3}{|c|}{$\begin{array}{l}\text { Tab. } 1 \\
\text { Estimation of savings during a five year Period 1962-1967 } \\
\text { (in Swedish Crowns) }\end{array}$} \\
\hline \multicolumn{3}{|l|}{ Savings } \\
\hline \multicolumn{3}{|l|}{$\begin{array}{l}\text { Increase in number of analyses about } \\
100 \% / 5 \text { years. This is estimated to } \\
\text { correspond to an increase in work load } \\
\text { of } 75 \% / 5 \text { years or } 12 \% / y e a r \text {. Salaries } \\
\text { for laboratory assistents and aids } \\
1962 / 631,200,000 \text {. }\end{array}$} \\
\hline $\begin{array}{l}\text { Estimated increase in salaries ( } 12 \% / \text { year }) \\
\text { during the } 5 \text { years period of not } \\
\text { automated }\end{array}$ & $2,496,000$ & \\
\hline $\begin{array}{l}\text { Staff increased about } 4 \% / \text { year. Corre- } \\
\text { sponds to a total increase in salaries } \\
\text { during these } 5 \text { years (adjusted to the } \\
\text { salary level of } 1962 / 63 \text { ) }\end{array}$ & $\underline{758,000}$ & $1,738,000$ \\
\hline Investments & & \\
\hline $\begin{array}{l}\text { 1. Automated analytical systems } \\
\text { 2. Computer time, rent for punches etc. }\end{array}$ & $\begin{array}{l}750,000 \\
550,000 \\
\end{array}$ & $1,300,000$ \\
\hline Gain & & 438,000 \\
\hline
\end{tabular}

for a chemical analysis, which is between three and four Swedish crowns, and is about the same price as that of the dispensible cannula and tube used in taking blood samples. An estimate of the saving obtained by introducing EDP and other measures to rationalize the laboratory work, is given in table 1 . In this table a saving of about 400,000 Swedish crowns has been estimated for a five year period. Consequently, it seems justifiable to state, that the system introduced has, at least, not increased the cost, despite the obvious medical and practical advantages.

\section{General conclusions}

The automated handling of data in a clinical chemical laboratory has significantly contributed (a) to increase the per capita working capacity by about 100 per cent in a fiver-year period, by reducing the clerical work to a minimum; and (b) to introduce a daily standardized quality control currently for about 400,000 determinations per year, representing about twentyfive analytical methods. The costs must be considered both acceptable and reasonable.

\title{
Referencecs
}

1. HJelar, M., W. Schneider, J. C. Vuille and G. Wallenius, Automated Acquisition, Processing and Communication of Laboratory Data in a Hospital. World Hospitals: l'Hospital le Monde 3, 174 (1967). - 2. HJELM, M., Quality Control of Automated Analytical Systems in Clincal Chemistry. This journal, to be published. - 3. CLS-Dataprocessing system for clinical chemical laboratories. Uppsala University Hospital, Uppsala Üniversity Datacenter, Uppsala, Sweden and IBM International Medical Support Center, Stockholm, Sweden (1968). - 4. Woob'ward, R. H. and P. L. GoldsMrth, Cumulative sum techniqués. I. C. I. Monograph No 3, Oliver and Boyd Ltd, Edinburgh and London (1964). University Hospital Uppsala, Sweden

Z. klin. Chem. u. klin. Biochem.

7. Jg., S. 100-104, Januar 1969

Round Table-Diskussion

\section{Round Table-Diskussion}

\author{
Bearbeitung: R. H. H. RICHTER, Bern
}

(Eingegangen am 25. November 1968)

Moderator: Prof. Dr. R. RichterICH, Bern

Teilnehmer: Dr. T. BRECHBÜHLER, Basel

PD Dr. Dr. H. Bütrner, Kiel

Dr. J. Bierens de HaAN, Genf
Dr. M. HJeLM, Uppsala

Dr. R. H. H. RICHTER, Bern

Dr. M. Rorh, Genf

PD Dr. Dr. D. Stamm, München

\section{Prof. Richterich}

Sehr geehrte Damen und Herren!

Ich habe keine Fragen erhalten; man könnte daraus entnehmen, daß heute nachmittag alles so klar war, daß für die gesamte Automation und Datenverarbeitung gar keine Probleme mehr übrigbleiben. Das zwingt mich, irgendwelche Fragen selbst hier zur Diskussion zu stellen, und ich möchte als erstes ein konkretes Problem diskutieren: Was macht man, wenn z. B. in Bern um
5 Uhr abends alle Labordaten im Rechenzentrum der Universität liegen und der Computer nicht fựktioniert? Ich möchte vielleicht Dr. Hjelm fragen. How do you solve the problem, when your computer breakes down?

\section{Dr. Hjelm}

A manual evaluation system is included in the system for this situation. This system consists of the recorders or digital printers, where the primary values always are registered before they enter into the data collecting unit. 
Ordinarily the recordings are used to check the runs, c. g. that high peaks correspond to high final patient values, that no irregularities are present and that the analogue values have been registered by the data collecting unit in duc time. The primary values for standard solutions, patient values and control samples can always be used, however, for manual calculations of final results. We think that this possibility is most important and necessary in a hospital despitc the fact that breakdowns in the data collecting unit or the computer have not happened more than two to three times a year during the last four-year period.

In case of a breakdown the statistical evaluation of the individual patient values has to be omitted. The accuracy of the runs can be judged by the control samples. If the data collecting unit is the missing part, we have to punch cards with the primary results, from the primary recordings to be able to store these results on disks. If the computer is the missing part, the primary results are already automatically punched on cards. Though these cards are not used for the immediate calculations they can be fed into the computer and stored on disks, as soon as the computer functions again.

\section{Prof. Richterich}

Möchte sich jemand sonst zu diesem Problem äußcrn? Bei uns geschicht es folgendermaßen: Wir sind natürlich viel weniger automatisiert als Dr. Hjelms Laboratorium. Wir führen die Mehrzahl der Analysen manuell durch und gehen mit diesen Daten in den Computer. Wir haben 2 Auswegslösungen, wenn der Computer am Rechenzentrum zusammenbricht. Zuerst gehen wir auf die Volksbank in Bern; diese verfügt über einen ähnlichen Computer, bei dem man mit einem adaptierten Programm durchkommt. Fällt dieser auch aus, dann müssen wir mit dem Auto nach Luzern. Ich glaube es ist außerordentlich wichtig, wenn man in einem Routinebetrieb Datenverarbeitung einführt, daß man von vornherein für jede auch noch so unwahrscheinliche Situation eine Lösung vorsicht.

\section{Dr. Büttner}

Es crgibt sich hier noch ein anderes Problem. Was passiert, wenn eine Analysenserie nicht in Ordnung gewesen ist? Was tun Sic dann im Eilfall, was tut Dr. Hjelm dann?

\section{Dr. Hjelm}

If the chemistry is down, the situation is analyzed from a chemical point of view. If the whole series has to be rerun and the analysis channel is connected to the data handling system and, off line, to the computer, the primary values stored in the computer are recalled. They are succeedingly replaced by the new, accurate values. Emergency samples can be given new, sequential numbers, if placed back in the series and, when the rerun starts, be immediately analyzed. If only some of the determinations of the series are inaccurate, the corre- sponding values in the computer are recalled, the samples are placed at the end of the series and are given new identification numbers. If the emergency samples are included they are handled as described above.

\section{Dr. Büttner}

How often do you have such troubles in your system?

\section{Dr. Hjelm}

On the average, if we consider just one channel: twice a month: and if we consider the system as such: twice a week.

\section{Prof. Richterich}

May I ask you something about your lecturc? Did I understand you correctly that you have about 5 qualified technicians and 30 uncertified personel?

\section{Dr. Hjelm}

We have 45 technicians.

\section{Prof. Richterich}

Sorry, then I have misunderstood you. But I think this discussion or your talk anyway has shown, that the current structure of a routine laboratory needs considerable change before data processing can be introduced in the laboratory.

Ich glaube, daß die ganze Struktur und Organisation unscrer Laboratorien ganz wesentlich verändert werden muß, bevor man überhaupt daran denken darf, Datenverarbeitung einzuführen. Wir haben da persönlich ziemlich große Probleme erlebt. Es würde mich sehr interessieren, was die anderen Herren zur Frage des Personals und der klassischen Laborstruktur denken.

\section{Dr. Büttner}

Da wir zur Zeit noch keine Datenverarbeitung haben, sondern uns im wesentlichen mit der Automation beschäftigen, möchte ich Ihre Frage im Hinblick auf die Automation beantworten. Dr. Hjelm sagte schon, daß er Elektronik-Ingenieure in seinem Laboratorium hat. Das ist an sich natürlich die ideale Lösung. Das haben wir nicht, aber wir haben immerhin einen Techniker, den wir in einigen Jahren auf die Automation eingearbeitet haben, und wir haben von fast allen Einzelteilen der Systeme Austauschgeräte. Wir können also, wenn irgend etwas versagt, austauschen. Das ist beim manuell arbeitenden Laboratorium nicht üblich. Und ich denke, daß, wenn man automatisiert, alle diese Dinge mitberücksichtigt werden müssen.

\section{Prof. Richterich}

Herr Stamm, möchten Sie sich auch noch dazu äußern?

\section{Dr. Stamm}

Wir sind jetzt gerade bei der Einführung der Datenverarbeitung in mehreren Stufen. Wir haben crst mit einem Kurs für die technischen Assistentinnen begonnen. $\mathrm{Da}$ 
stimme ich eben mit Ihnen überein, Herr Richterich, daß man für das technische Personal die Datenerfassung und Verarbeitung weitgehend verständlich und durchsichtig machen muß.

\section{Dr. Bierens de Haan}

Il me semble qu'il y a une autre remarque intéressante à faire, c'est qu'il y a de bonnes et de mauvaises automatisations (ou mécanisations) des analyses. MM. Büttner et Hjelm ont longuement expliqué comment on utilise les ordinateurs pour récupérer, en quelque sorte, des appareils imparfaits ... J'y reviens, mais il est très frappant de voir à quel point vos exposés sont venus appuyer le mien, sur ce point. Une panne d'ordinateur, lorsque celui-ci est occupé à contrôler, à surveiller, à corriger des autoanalyseurs est beaucoup plus catastrophique que lorsqu'il est branché sur des appareils simples à sortie digitale directe, c'est à dire sans même l'aide de convertisseurs analogiques digitaux, comme on peut les faire maintenant.

N'assiste-t-on pas à une hypertrophie du traitement de l'information, pour des machines qui sont mauvaises?

\section{Prof. Richterich}

I think, extremely formulated, Dr. de Haan meant that you can use a photometer costing SFr. 50.- and hook it up to a computer and still get correct results because you are taking care of all the faults of the primary instruments by means of the computer.

\section{Dr. Hjelm}

Well, I guess, that one can always connect an instrument to a data collecting system, to mechanize the registration of analogue values from it as the quality of the registered primary values will be the same in manual and automated registration systems. In fact, much time can be saved, however, by mechanizing the data collecting part of the laboratory work.

In my opinion, it is another question, to what the primary values should be corrected. This must, at present, probably be evaluated mainly from a clinical point of view, i. e. the results must have reliability which corresponds to the actual clinical situation. That means that the final treatment of the primary values in the computer should be as simple as possible. I agree completely, that the weakest link of automated laboratory systems with automated data acquisition and data handling is the analytical instruments and that the behaviour of the devices, now present on the market, will not, as such, improve by being connected to a computer. This is also one of the reasons why we have decided to use an "offline" system for our present instruments, though we certainly realize that "on-line" systems must be the final solution.

\section{Prof. Richterich}

Diese Frage, daß es ziemlich bald oder sagen wir konkret in den nächsten 5 Jahren, möglich sein wird, alles, alle
Methoden so zu automatisieren, daß man direkt, also über Analog-Digital-Umwandlung in den Computer gehen kann, wird in Schweden überwiegend positiv beantwortet. Ist das richtig?

\section{Dr. Hjelm}

The trend is to automate the voluminous work load, i. e. $40-50$ methods, probably corresponding to $80-90$ per cent of all analyses per year and to try to store these results and also results from manual methods, at present about 100 , on disks, tape etc., to enable e. g. the production of cumulative reports on patients, including all types of chemical tests.

\section{Prof. Richterich}

Das ist die skandinavische Auffassung; sie variiert ziemlich weitgehend von dem, was wir bisher hier in Mitteleuropa gemacht haben, wo die wenigsten Leute eine so moderne und fortschrittliche Auffassung vertreten. Ich bin nicht ganz überzeugt, ob das wirklich die definitive Lösung sein wird, und ich glaube. Dr. de Haan hat Beispiele erzählt. Sprechen wir einmal ganz konkret von bestimmten Methoden, nehmen wir die Flammenphotometrie von Natrium/Kalium; da hat man die Alternative auf einen 4-Channel Autoanalyzer zu gehen, - oder 12-Channel, das spielt keine Rolle - oder man kann mit einem modernen Instrument, das nach einer einzigen Verdünnung Natrium und Kalium ańzeigt, direkt ablesen, wobei es durchaus kein Problem wäre, nun diese Digitalanzeige direkt in den Computer zu geben. Hier ist aber immerhin noch ein ganz wesentlicher manueller Schritt dabei, nämlich die Tatsache, daß jemand, eine Laborantin, vor diesem Gerät sitzt, die Probe nimmt, zum mindesten in einen Probenwechsler stellt, vielleicht von Hand noch verdünnt und dafür sorgt, daß die Identifizierung richtig erfolgt.

\section{Dr. Büttner}

Wir haben vor einiger Zeit vor der Frage gestanden: sollten wir mit den Elektrolyten auf den Aưtoanalyzer gehen oder nicht. Damals war das Technicon Flammenphotometer noch nicht auf dem Markt. Mit dem ILFlammenphotometer kann die Analyse einschließlich Verdünnung vom Serum bis zur Digitalausgabe Natrium/ Kalium gleichzeitig in 10 Sekunden durchgeführt werden. Es ist genau so schnell wie der Autoanalyzer. Aber Sie haben noch einen manuellen Schritt dazwischen: Sie müssen das Röhrchen noch unter den Dilutor halten. Alles andere ist praktisch auch mechanisiert, aber diese Zwischenlösung bedeutet für uns, daß die. Präzision unserer Analysen beim Natrium etwas über 1\% liegt und nicht wie beim Autoanalyzer zwischen 4 und $5 \%$ und noch schlechter.

\section{Prof. Richterich}

Ja, dies bringt uns eigentlich zur Kritik der Automaten. Es würde mich vielleicht doch noch interessieren, was die Meinung der anderen Herren zur Frage ist, und nun bitte ich um Ihre ganz private, persönliche Meinung: 
Kommen wir in den nächsten 10 Jahren zum vollständig automatischen Labor in einer Konzeption, wie sie Herr Hjelm dargestellt hat? Oder kommen wir im großen Routinebetrieb zum vollständigen Anhängen aller Apparate an einen laborinternen Computer oder glauben Sie an Zwischenlösungen, an Halb-Automaten und ähnliches?

Dr. Roth, was meinen Sie dazu?

\section{Dr. Roth}

Je crois que nous sommes dans une phase où l'on doit encore développer la simplification de certaines méthodes analytiques. Un des moyens consiste à éliminer certaines opérations qui font perdre du temps, telles que la déprotéination; il y a de plus en plus de méthodes qui permettent de travailler sur des sérums complets et cettc simplification nous conduit à des méthodes extrêmement rapides. On a parlé de la photométrie de flamme. Il s'agit d'une méthode si simple, permettant de faire des analyses à unc cadence tellement rapide, que l'automation n'est plus nécessaire. Est-ce qu'il faut renoncer entièrement au personnel? Je ne pense pas que ça soit absolument nćcessaire; il y aura toujours des gens dans les laboratoircs et il faut aussi poser la question du prix. Dans certains cas le personnel n'est pas si cher, si l'on compare son coût à celui de certains automates qui doivent être amortis en quclques années pour faire des opérations automatiques qui pourraient aussi se fairc très rapidement à la main. Il y a là un choix à faire entre le travail manuel rapide et simple, assisté par des appareils semiautomatiques et l'automation complète.

\section{Dr. Brechbühler}

Auch ein anderes Problem stellt sich bei allen Automatisierungen. Dieses liegt nicht in der Auswertung und Datenverarbeitung, sondern die Schwierigkeiten sind bedingt durch die oft geringen zur Verfügung stehenden Probemengen, z. B. bei Kindern. Es wurde heute morgen ja auch wieder gezeigt, was für Probleme in bezug auf Empfindlichkeit, Lichtausbeute usw., schon eine einfache photometrische Messung aufgeben kann, bis man schließlich ein richtiges Resultat erhält. Für die nächste Zukunft wird es sicher nicht zu einer alles umfassenden Vollautomation kommen, vielmehr werden in gewissen Spitälern und bei bestimmten Analysen höchst wahrscheinlich $Z$ wischenlösungen gewählt werden, und z. B. Verdünnungsautomaten, integrierende Photometer, kombiniert mit manuellen Methoden, werden immer noch gebraucht werden.

\section{Prof. Richterich}

Das ist zweifellos richtig. Das Problem wird immer bleiben, und ich würde noch weiter gehen und sagen, daß wahrscheinlich bei uns die Notfallanalysen für die nächsten 10 Jahre nicht über den Computer ausgegeben werden, sondern daß die Probe ins Labor kommt, die Analyse manucll oder teilweise automatisiert durch- geführt wird und das Resultat dann telefonisch so schnell wic möglich - denn da geht es ja um Minuten - an die Abteilung - vielleicht über Teleprint oder irgend etwas Ähnliches - ausgegeben wird. Aber dic letzten paar Min. möchten wir noch darauf verwenden, um kurz auf dic finanziellen Aspekte cinzugehen; denn da kann man nun die widersprechendsten Meinungen hören. $\mathrm{Da}$ wird Ihnen ein Vertreter ins Haus kommen und sagen: Kaufen Sic diesen Computer für 2 Millionen Franken, und in 5 Jahren sind Sic aus den Ersparnissen, dic Sie in der Zwischenzeit gemacht haben, ein reicher Mann geworden. Wenn Sic dann ein Gesuch an dic Regierung stellen, dann heißt es: stellen Sic licber noch ein paar Leute mehr ein, es kommt immer noch billiger als wenn Sie einen Computer verwenden. Und da muß man versuchen, nach Mittelwegen zu suchen. Die großzügigsten Wege haben ganz zweifellos die skandinavischen I_änder, ganz speziell Schweden, eingeschlagen, die nun mit rein nationalökonomischen Methoden an dieses Problem herangetreten sind und so rechnen wic eine Großfirma. Sie betrachten das Laboratorium nicht mehr einfach als Dienstlcistungsbetrieb im Spital, sondern als Bctricb im eigentlichen Sinne des Wortes; dies ist letzten Endes sicher der einzig vernünftige ,approach“.

Wie ist die Situation in Deutschland, Herr Büttner?

\section{Dr. Büttner}

$\mathrm{Da}$ ist die Situation im großen und ganzen ähnlich. Aber es sind doch eine Reihe von psychologischen Widerständen bei der Einführung der Datenverarbeitung im Bereich der Klinik zu überwinden. Ich glaube, das ist auch ein wesentlicher Punkt. Vielleicht eine ganz spezielle Frage noch, die ja auch ein finanzielles Problem berührt: Kleiner Laborcomputer oder großer Computer in einem Zentrum? Dic Frage wird in der nächsten Zeit für viele große Laboratorien kommen, wo einerseits geplant wird, einen Klinik-Computer für das ganze Klinikum einzurichten und anderseits eben die Alternative besteht, einen kleinen Computer, der etwa 200000-300000 DM kostet, im Labor zu installieren. Ich möchte meinen, daß die letztere Lösung augenblicklich die bessere ist.

\section{Prof. Richterich}

Ich würde dies persönlich bezweifeln. Ich wollte das auch vor 5 Jahren. Ich habe dann ein Gesuch an die Regierung gemacht und erhielt den Bescheid, daß dies überhaupt nicht in Frage komme, und zwar deshalb nicht, weil - ich glaube bereits 1956 - der Kanton Bern beschlossen hat, daß öffentliche Anstalten keine cigenen Computer erhalten, sondern daß die Gesuche an eine zentrale kantonale Rechenstelle gehen müssen, die dann möglichst zentralistische Lösungen sucht. Wir haben eine sehr befriedigende Lösung gefunden. Wir haben nämlich vom Spital einen Mathematiker zur Verfügung gestellt bekommen sowic jetzt einen Programmierer, und dafür hat sich das kantonale Rechenzentrum verpflichtet, alle Spitalarbeiten mit Priorität durchzuführen. Also 
Punkt 5 Uhr, wenn unsere Lochkarten kommen, wird jede andere Arbeit unterbrochen, und unser Material ist 5.15 Uhr verarbeitet. Für unsere lokalen Berner Verhältnisse würde ich unbedingt dem zentralen GroßComputer mit dem hochspezialisierten Personal, mit dem ständigen Service den Vorzug geben. Ich wäre heute geradezu unglücklich, wenn ich einen eigenen Computer im Labor aufstellen müßte.

Dr. Hjelm would you mind to explain your calculations. An dieser Stelle sprach Dr. Hjelm über seine Schätzung der Ersparnisse einer 5 Jahres-Periode durch Einführung der Datenverarbeitung; dieser Bericht wurde dann später ins Manuskript von Dr. Hjelm aufgenommen.

\section{Prof. Richterich}

Ich bin gleicher Meinung wie Herr Hjelm und bin froh, $\mathrm{da} ß$ er dies hier so klar gesagt hat. Ich glaube, es ist sehr wichtig, daß wir anfangen, innerhalb unserer Labor- betriebe, die mehr und mehr industrielle Ausmaße annehmen, nach industriellen Grundsätzen zu denken.

Damit möchte ich schließen. Ich möchte vor allem Herrn Hjelm herzlich danken, der von Uppsala extra nach Basel gekommen ist, um uns dieses außerordentlich aufschlußreiche Referat zu geben. Ich hatte das Vergnügen, ihn vor 2 oder 3 Jahren kurz zu besuchen. Ich war damals schon hell begeistert von der Art, wie mit logischer Methode an das schwierige Problem herangegangen wurde.

Ich möchte aber auch unseren deutschen Kollegen, Herrn Büttner und Herrn Stamm, herzlich danken und selbstverständlich auch allen Schweizern, die hier anwesend sind. A uch Ihnen, daß Sie so lange gewartet haben.

Die Tagung wurde in vorzüglicher Weise von Herrn Dr. phil. Max Keller, Leiter des Laboratoriums des Frauenspitals Basel, organisiert. 